\title{
The Expression of Human Telomerase Reverse Transcriptase in Adult Acute Myeloid Leukemia and Its Correlation With Various Clinico-Pathological Parameters
}

\author{
Farah AlJobori $^{1} \&$ Abdulkareem Mohammad Jaafar ${ }^{1}$ \\ ${ }^{1}$ Department of Pathology, College of Medicine, University of Baghdad, Iraq \\ Correspondence: Farah Abdulmahdi AlJobori, Department of Pathology, College of Medicine, University of \\ Baghdad, Iraq.
}

Received: January 13, 2019 Accepted: February 22, 2019 Online Published: March 12, 2019

doi:10.5539/gjhs.v11n4p25 URL: https://doi.org/10.5539/gjhs.v11n4p25

\begin{abstract}
Background: Acute myeloid leukemia (AML) is a heterogeneous disorder characterized by clonal expansion of myeloid progenitors (blasts) in the bone marrow and peripheral blood, AML accounts for $80 \%$ of acute leukemia in adults, its incidence increase with age. AML can be a fatal disease so research to predict prognosis is important. Telomerase (TA) is an enzyme that stabilizes the telomere length and makes the cell immortal. It is present in some of the normal cells, fetal cells, adult germ cells, and presents in $85 \%$ of tumors in humans, it has been shown that TA can be used as a prognostic marker in some solid and hematological neoplasms. Telomere length is a factor that predicts telomere function.
\end{abstract}

Aim: We test the quantitative amount of human telomerase reverse transcriptase (hTERT) gene expression in AML (diagnosed according to FAB) adult and its correlation with various clinic-pathological parameters.

Patients and Methods: We used the TRAP assay to assess the hTERT gene expression in mononuclear blood cells from 40 newly diagnosed AML patients, 25 AML patients after completing their course of treatment, and 15 control health subjects.

Results: The mean value of hTERT in AML and control groups were $[1.59 \pm 1.27 \mathrm{anm}$ and $0.035 \pm 0.046 \mathrm{anm}$ respectively], and this difference was significantly higher in patients than in control group $(p=0.0001)$. The telomerase activity was positive in 27 (67.5\%) AML patients, while 13 (32.5\%) AML patients were negative for telomerase activity. Twenty-five patients after induction chemotherapy were followed up by bone marrow and peripheral blood examination to determine the patient's response to therapy. Complete hematological remission was achieved in $12(48.0 \%)$ patients and incomplete hematological remission in $13(52.0 \%)$ patients (14\%). The hTERT level was significantly higher in patients before induction chemotherapy than after completion of the induction course $(\mathrm{p}=0.0001)$. The hTERT level at diagnosis in patients who did not achieve complete hematological remission was significantly higher than that in patients who achieved complete hematological remission $(\mathrm{p}==0.026)$. The hTERT level after induction therapy was significantly higher in patients who did not achieve complete hematological remission than in patients who achieved complete hematological remission $(\mathrm{p}=$ $0.003)$.

Conclusion: Our research suggests that the hTERT expression could serve as a prognostic marker for AML patients.

Keywords: AML, telomerase, hTERT, prognosis, TRAP assay

\section{Introduction}

Acute myeloid leukemia (AML) is a cancerous disease of the bone marrow that hematopoietic precursors are trapped in the early stage of development. The AML subtypes are distinguished from other related blood disorders by the presence of more than $20 \%$ bone marrow blasts (Ghiaur, Wroblewski, \& Loges, 2015). The arrest of bone marrow cells in early stages of maturation is under research, but in many situations, it involves the activation or inactivation of genes into chromosomal translocations and other genetic and/or epigenetic abnormalities (Arber et al., 2016).

Telomerase is a reverse transcriptase enzyme that carries its own RNA molecule, which is used as a template when 
it elongates telomeres. Telomerase is active in normal stem cells and most cancer cells but is normally absent from, or at very low levels in, most somatic cells (Shay, 2016).

Telomerase reverse transcriptase is a catalytic subunit of the enzyme telomerase, and its function is to lengthening the DNA strands of telomeres (Skvortsov. Zvereva, Shpanchenko, \& Dontsova, 2011).

Telomerase activity and hTERT appears as a key event in the development and progression of the many diseases, whereas in acute myeloid leukemia (AML), in chronic myeloid leukemia (CML) and in B-cell diseases, it was demonstrated that telomerase activity is not required for the initiation of disease, but it is required for its maintenance (Ropio, Merlio, Soares, \& Chevret, 2016).

\section{Method}

A prospective cross-sectional study conducted on forty adult patients with newly diagnosed de novo AML according to the laboratory and flow-cytometric criteria and follow up was performed for twenty-five patients after one month of induction therapy. The collection period is from November 2017 to October 2018. The patients were seen in the Hematology Unit of Baghdad Teaching Hospital in Medical City.

The ethical committee of the College of Medicine, University of Baghdad, approved this study. Informed consents were taken from all participants.

Inclusion Criteria

1). Patients enrolled in the study after their diagnosis as AML in the ward.

2). All AML patients were above 15 years old.

3). All AML patients were newly diagnosed, and they were not receiving any chemotherapy before the time of collecting blood and BM samples.

Exclusion Criteria:

1). Patients with a history of myelodysplastic syndrome or therapy-related secondary AML cases.

2). Patients who died during induction therapy.

3). Cases with dry tap due to bone marrow fibrosis.

\subsection{Therapy and Follow-Up}

Twenty-five patients after induction chemotherapy were followed up by bone marrow and peripheral blood examination to determine the patient's response to therapy. Patients were treated according to the standard chemotherapy protocol of AML. After induction chemotherapy, patients were followed up by CBC and bone marrow examination to determine the patient's response to therapy.

Patients were classified into:

I. Complete remission.

1). Less than $5 \%$ blasts in an aspirate sample with marrow spicules and with a count of at least 200 nucleated cells, there should be no blasts with Auer rods.

2). Peripheral neutrophil count of more than $1.5 \times 10^{9} / \mathrm{L}$.

3). Platelet count above $100 \times 10^{9} / \mathrm{L}$.

4). Absence of extramedullary disease

II. Incomplete remission: the patient who did not achieve complete hematological remission.

\subsection{Method}

Repeated Amplification Protocol (TRAP) method was used to detect the hTERT telomerase activity (Mender, \& Shay, 2015). Utilizing the Telomeric Repeat Amplification Protocol was done by photometric enzyme immunoassay kit (Telo TAGGG Telomerase PCR ELISA), developed by Roche applied science. Three ml of fresh peripheral blood were collected from patients as well as the control group to do the test. By subtracting the mean of absorbance readings of the negative controls from those of samples. If the difference is higher than 0.2 , this will consider positive for hTERT.

\subsection{Statistical Analysis}

Statistical analysis was performed with the IBM-SPSS 23 statistical software program. Associations between categorical variables were assessed via cross-tabulation and chi-square test. Mann-Whitney test (a non-parametric 
equivalent of the independent samples t-test), Wilcoxon signed rank test (a non-parametric equivalent of the paired t-test) and Kruskal-Wallis test (a non-parametric equivalent to one-way ANOVA) were used to compare means of continuous variables. Exact tests were used to calculate the p-value. In all statistical analyses, a p-value $<0.05$ was considered significant.

\section{Results}

The current study included forty patients with acute myeloid leukemia, and fifteen age and sex-matched healthy individuals ( 8 males and 7 females with a mean age of $41.47 \pm 13.55$ years) as a control group. The age of AML patients ranged between 15 and 84 years with a mean age of $40.13( \pm 17.62)$ years. Fifteen $(37.5 \%)$ patients were males, and twenty-five $(62.5 \%)$ were females. The male to female ratio was 0.6. For risk stratification, AML patients included eight $(20.0 \%)$ patients aged $\geq 60$ years and thirty-two $(80.0 \%)$ patients aged $<60$ years.

According to the French-American-British (FAB) classification system using morphological, cytochemical and immunophenotypic criteria, there were 11 (27.5\%) patients diagnosed with AML-M3, $8(20 \%)$ patients diagnosed with AML-M2, 7 (17.5\%) patients diagnosed with AML-M4, 5 (12.5\%) patients diagnosed with AML-M0 and same for M1, and $4(10 \%)$ patients diagnosed with AML-M5. The hematological parameters of the forty AML patients are summarized in Table 1.

Table 1. Hematological characteristics of AML patients

\begin{tabular}{lll}
\hline $\begin{array}{l}\text { Hematological parameter } \\
(\mathrm{N}=40)\end{array}$ & Range & Mean $\pm \mathrm{SD}$ \\
\hline $\mathrm{Hb}(\mathrm{gm} / \mathrm{dl})$ & $3.30-14.3$ & $7.74 \pm 2.39$ \\
Total WBC count $\left(10^{3} / \mu \mathrm{l}\right)$ & $0.6-14.04$ & $5.76 \pm 3.76$ \\
Neutrophil count $(103 / \mu \mathrm{l})$ & $0.3-7.00$ & $2.87 \pm 1.93$ \\
Platelet count $\left(10^{3} / \mu \mathrm{l}\right)$ & $14-378$ & $80.03 \pm 69.42$ \\
Blast $\%$ (Bone marrow) & $35-97$ & $66.43 \pm 18.07$ \\
\hline
\end{tabular}

\section{1 hTERT Level and Telomerase Activity}

The hTERT level in the AML patients was ranged between 0.010 and 3.710 (anm), while in the control group it ranged between 0.000 and $0.11(\mathrm{anm})$. The mean value of hTERT in AML and control groups were [1.59 \pm 1.27 (anm) and $0.035 \pm 0.046(\mathrm{anm})$ respectively], and this difference was significantly higher in patients than in control group $(\mathrm{p}=0.0001)$. In addition, the telomerase activity was positive in $27(67.5 \%)$ AML patients, while $13(32.5 \%)$ AML patients were negative for telomerase activity.

Twenty-five patients after induction chemotherapy were followed up by bone marrow and peripheral blood examination to determine the patient's response to therapy. Complete hematological remission was achieved in 12 (48.0\%) patients and incomplete hematological remission in $13(52.0 \%)$ patients (14\%).

The hTERT level was significantly higher in patients before induction chemotherapy than after completion of the induction course $(\mathrm{p}=0.0001)$, Table 2 .

Table 2. Comparison of hTERT level between patients before and after Treatment

\begin{tabular}{llll}
\hline & Groups & & Pvalue \\
\hline & Patients before treatment & Patients after treatment & \\
$\mathrm{N}=25$ & $\mathrm{~N}=25$ & \\
& Mean $\pm \mathrm{SD}$ & Mean $\pm \mathrm{SD}$ & $0.0001^{*}$ \\
\hline hTERT level (anm) & $2.022 \pm 1.207$ & $0.759 \pm 1.113$ & \\
\hline
\end{tabular}

The hTERT level at diagnosis in patients who did not achieve complete hematological remission was significantly higher than that in patients who achieved complete hematological remission $(\mathrm{p}=0.026)$. Also, at diagnosis, a significantly higher proportion of patients who had incomplete hematological remission $(65.0 \%)$ were associated 
with positive telomerase activity than the patients with complete response $(35.0 \%)(\mathrm{p}=0.015)$, table 3 .

The hTERT level after induction therapy was significantly higher in patients who did not achieve complete hematological remission than in patients who achieved complete hematological remission $(\mathrm{p}=0.003)$.

After completion of induction chemotherapy, a significantly higher proportion of patients who had incomplete hematological remission $(87.5 \%)$ were associated with positive telomerase activity than the patients with complete response $(12.5 \%)(\mathrm{p}=0.03)$.

Table 3. Associations between telomerase activity before treatment and hematological response

\begin{tabular}{|c|c|c|c|c|}
\hline & & \multicolumn{2}{|c|}{ Hematological response } & \multirow[b]{2}{*}{ Total } \\
\hline & & Complete response & Incomplete response & \\
\hline \multirow[t]{4}{*}{ Telomerase activity (Before treatment) } & Positive & 7 & 13 & 20 \\
\hline & & $35.0 \%$ & $65.0 \%$ & $100.0 \%$ \\
\hline & Negative & 5 & 0 & 5 \\
\hline & & $100.0 \%$ & $0.0 \%$ & $100.0 \%$ \\
\hline \multirow[t]{2}{*}{ Total } & & 12 & 13 & 25 \\
\hline & & $48.0 \%$ & $52.0 \%$ & $100.0 \%$ \\
\hline
\end{tabular}

$p=0.015^{*}$.

Receiver operating characteristic (ROC) curve was used to calculate a cut off value for the hTERT level at diagnosis (Figure 1)

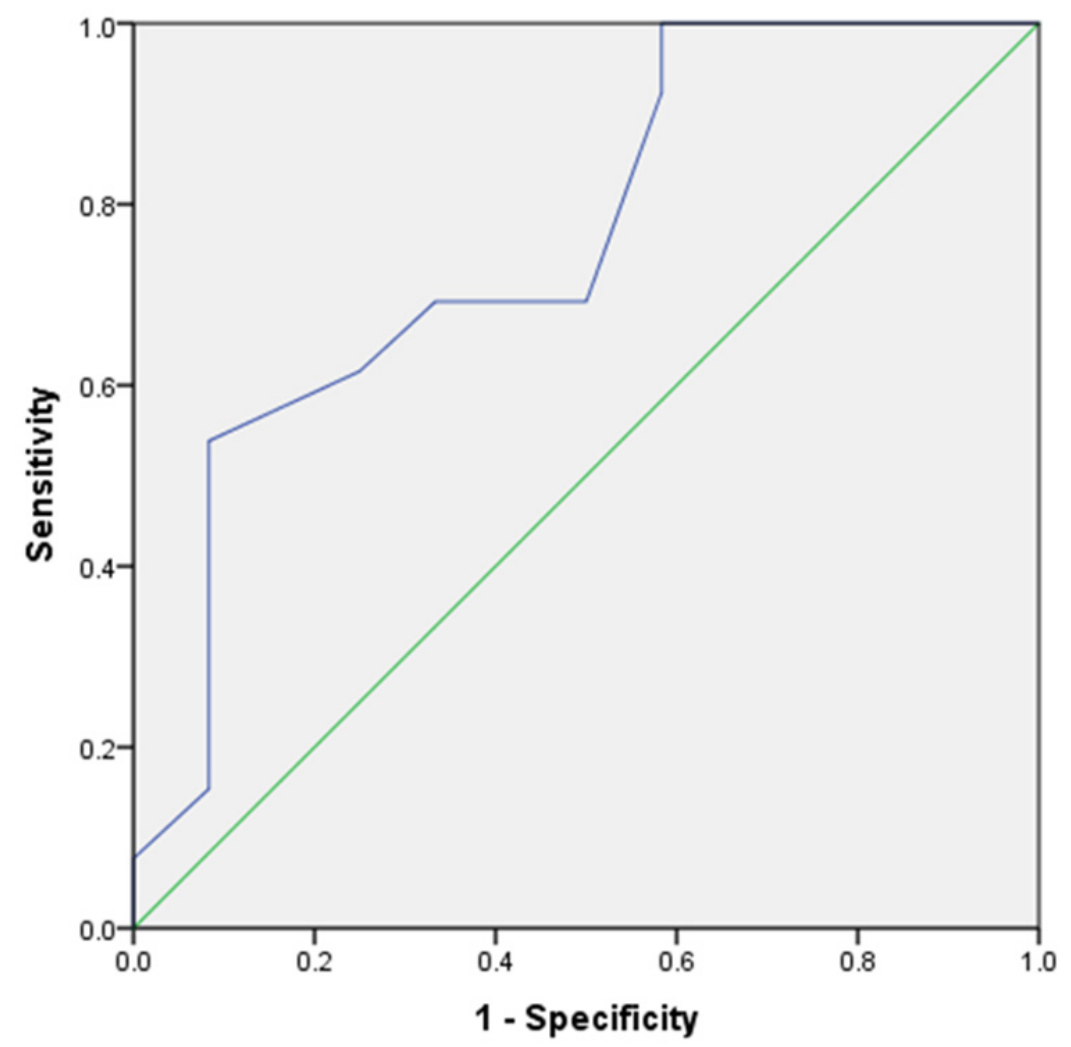

Figure 1. ROC curve showing cut off value of $2.01 \mathrm{anm}$ for hTERT level as a predictive value for AML outcome after induction chemotherapy 
The hTERT level after induction therapy was significantly higher in patients who did not achieve complete hematological remission than in patients who achieved complete hematological remission $(p=0.003$, Table 4).

Table 4. Comparison of hTERT level after treatment according to hematological response

\begin{tabular}{lll}
\hline & Hematological response & \\
\cline { 2 - 3 } & Complete & Incomplete \\
& $\mathrm{N}=12$ & $\mathrm{~N}=13$ \\
& Mean $\pm \mathrm{SD}$ & Mean $\pm \mathrm{SD}$ \\
\hline hTERT level after treatment (anm) & $0.2533 \pm 0.71221$ & $1.2254 \pm 1.23413$ \\
\hline
\end{tabular}

$p=0.003 *$.

\section{Discussion}

Acute Myeloid Leukemia is the commonest acute leukemia in adult (Howlader et al., 2017). It represents the third most common primary cancer in Iraq (Iraqi cancer registry, 2011). The response to treatment in AML and survival is heterogeneous and so researches done to evaluate prognostic factors like age, karyotype, and general condition.

In this study, we try to assess the role of telomerase activity in AML and its potentiality as a prognostic factor.

One of the important prognostic factors in AML is age (Bernasconi et al., 2012). The mean age of our patients was 40.13 ( \pm 17.62$)$ years. This finding was comparable with other Iraqi studies (Almohsen \& Al-Mudallal, 2014), while in developed countries the mean age tend to be higher than this study and the majority of the patients are above 55 years old (Howlader et al., 2017), (Ayesh, Khassawneh, Matalkah, Alawneh, \& Jaradat, 2012). This difference may be related to the higher mean age of western population than the Iraqi population.

The hTERT level was significantly higher in patients than in control group. This finding goes with other previous studies that reported increased hTERT level in different solid tumors (Herbert, Wright, \& Shay, 2001), and hematological malignancies (Hartmann et al., 2005), (AlJabban \& Alalsaidissa, 2018), (Wang et al., 2014).

Since many malignant neoplasms showed increased hTERT activity, it may contribute to human carcinogenesis, as it results in increased telomerase activity (Jordanides et al., 2006).

In the current study, it was shown that hTERT level at diagnosis was significantly higher in patients who did not achieve complete hematological response than patients who achieved a complete hematological response. This finding suggests that hTERT level may act as a prognostic marker for the outcome of treatment. This phenomenon can be explained by a decrease in leukemic cells that synthesize hTERT and the emerging of the new healthy cell with low or absent telomerase activity (hTERT) in the bone marrow and the blood of patients who achieved complete remission (Jordanides et al., 2006), (Engelhardt, Mackenzie, Drullinsky, \& Silver, 2000).

In our study, it showed that the hTERT associated with AML and contribute and predict the response of AML patients to treatment, Also the research was done in our department by AlJabban (AlJabban \& Alalsaidissa, 2018) measuring the hTERT in CLL patients and its prediction of patients prognosis and their results were associated with patients prognosis and a lot of comparison between our results and AlJabban results, this gives us strong evidence that hTERT associated with hematological malignancies and it could play an important role in Iraqi patients prognosis that had hematological malignancies. Also, the telomere and telomerase relations appear to be a vital factor for proliferative abilities of tumor cells. It has been known that telomerase activity offers the capability of proliferation to the malignant cell; thus, targeting of tumor cells by hindering telomerase may be an effective therapy.

Also, receiver operating characteristic (ROC) curve was used to calculate a cut off value for the hTERT level at diagnosis. The Area under the curve $(\mathrm{AUC})=0.760$ (overall accuracy $=76.0 \%$ ). This finding indicates that, in AML patients with a value less or equal to $2.01 \mathrm{anm}$ hTERT level, $76.0 \%$ of patients will achieve complete remission (sensitivity [true positive cases] was $69.2 \%$, and specificity [true negative cases] was $66.7 \%$ ) (El Belbesy et al., 2015).

\section{Conclusion}

1) The hTERT level at diagnosis was significantly lower in patients who achieved complete hematological response than patients who did not achieve a complete hematological response. This finding suggests that 
hTERT level may act as a prognostic marker for the outcome of treatment.

2) The hTERT level may be used as prognostic marker for hematological malignancies.

\section{Acknowledgments}

We would like to acknowledge Dr.Ali AlJabban for his contribution in proofing the manuscript of this paper.

\section{Competing Interests Statement}

The authors declare that there are no competing or potential conflicts of interest.

\section{References}

Aljabban, A., \& Alalsaidissa, J. (2018). The Expression of Human Telomerase Reverse Transcriptase Gene and Its Activity in Patients with B-Cell Chronic Lymphocytic Leukemia and Its Impact on Clinical Staging. Global Journal of Health Science, 10(5). 167. https://doi.org/10.5539/gjhs.v10n5p167

Almohsen, F. S., \& Al-Mudallal, S. S. (2014). Relationship between the Expression of CD34, CD123 and Myeloperoxidase Markers by Flow Cytometry and Response to Induction Therapy in Acute Myeloid Leukemia. Iraqi JMS 2014.

Arber, D. A., Orazi, A., Hasserjian, R., Thiele, J., Borowitz, M. J., Le Beau, M. M., ... \& Vardiman, J. W. (2016). The 2016 revision to the World Health Organization classification of myeloid neoplasms and acute leukemia. Blood, 127(20), 2391-405. https://doi.org/10.1182/blood-2016-03-643544

Ayesh, M., Khassawneh, B., Matalkah, I., Alawneh, K., \& Jaradat, S. (2012). Cytogenetic and morphological analysis of de novo acute myeloid leukemia in adults: a single center study in Jordan. Balkan Journal of medical genetics: $B J M G, 15(1), 5-10$. https://doi.org/10.2478/v10034-012-0001-3

Bernasconi, P., Klersy, C., Rocca, B., Calvello, C., Orlando, A., Boni, M., ... \& Zappasodi, P. (2012). Human Telomerase Reverse Transcriptase (hTERT): A New Potential Prognostic Marker in AML?. Blood Nov, $120(21), 4790$.

Cong, Y. S., Wright, W. E., \& Shay, J. W. (2002). Human telomerase and its regulation. Microbiology and molecular biology reviews: MMBR, 66(3), 407-25, table of contents. https://doi.org/10.1128/MMBR.66.3.407-425.2002

Engelhardt, M., Mackenzie, K., Drullinsky, P., \& Silver, R. (2000). Telomerase activity and telomere length in acute and chronic leukemia, pre and post-ex vivo culture. Cancer Res, 60(13), 610-617.

Ghiaur, G., Wroblewski, M., \& Loges, S. (2015) Acute Myelogenous Leukemia and its Microenvironment: A Molecular Conversation. Semin Hematol, 52(3), 200-6. https://doi.org/10.1053/j.seminhematol.2015.03.003

Hartmann, U., Brummendorf, T. H., Balabanov, S., Thiede, C., Illme, T., \& Schaich, M. (2005). Telomere length and hTERT expression in patients with acute myeloid leukemia correlates with chromosomal abnormalities. Haematologica, 90, 307-316.

Herbert, B. S., Wright, W. E., \& Shay, J. W. (2001). Telomerase and breast cancer. Breast cancer research: BCR, 3(3), 146-9. https://doi.org/10.1186/bcr288

Howlader, N., Noone, A. M., Krapcho, M., Miller, D., Bishop, K., Kosary, C. L., ... Cronin, K. A.(2017). SEER Cancer Statistics Review, 1975-2014, National Cancer Institute. Bethesda, MD, https://seer.cancer.gov/csr/1975_2014/, based on November 2016 SEER data submission, posted to the SEER web site, April 2017.

Iraqi cancer registry: Leukemia statistic of the year 2011. Ministry of Health /Iraqi Cancer Board.2011.

Jordanides, N. E., Keith, W. N., Hills, R. K., Wheatley, K., Luong, Q. T., Burnett, A. K., ... \& Drummond, M. W. (2006). htert expression in Aml is heterogeneous and is of likely prognostic significance: 115. British Journal of Haematology, 133, 63. https://doi.org/10.1111/j.1365-2141.2006.06027.x

Mender, I., \& Shay, J. W. (2015). Telomerase Repeated Amplification Protocol (TRAP). Bio-protocol, 5(22), e1657. https://doi.org/10.21769/BioProtoc.1657

Ropio, J., Merlio, J. P., Soares, P., \& Chevret, E. (2016). Telomerase Activation in Hematological Malignancies. Genes, 7(9), 61. https://doi.org/10.3390/genes7090061

El Belbesy, S. F., El Aggan, H. A., Sultan, H. K., El Naggar, A. A., \& Ahmed, H. K. F. (2015). Telomere length and human telomerase reverse transcriptase (hTERT) level in patients with acute myeloid leukemia: Impact on clinical outcome. Acta Haematologica Polonica, 46(4), 304-311. 
https://doi.org/10.1016/j.achaem.2015.07.003

Shay, J. W. (2016). Role of Telomeres and Telomerase in Aging and Cancer. Cancer Discov, 6(6), 584-93. https://doi.org/10.1158/2159-8290.CD-16-0062

Skvortsov, D. A., Zvereva, M. E., Shpanchenko, O. V., \& Dontsova, O. A. (2011). Assays for detection of telomerase activity. Acta Naturae, 3(1), 48-68.

Wang, L., Xiao, H., Zhang, X., Wang, C., \& Huang, H. (2014). The role of telomeres and telomerase in hematologic malignancies and hematopoietic stem cell transplantation. Journal of hematology \& oncology, 7(1), 61. https://doi.org/10.1186/s13045-014-0061-9

\section{Copyrights}

Copyright for this article is retained by the author(s), with first publication rights granted to the journal.

This is an open-access article distributed under the terms and conditions of the Creative Commons Attribution license (http://creativecommons.org/licenses/by/4.0/). 